\author{
DEPARTMENT OF MATHEMATICS \\ UNIVERSITY OF NIJMEGEN The Netherlands
}

\title{
ASYMPTOTIC INFERENCE FOR NEARLY UNSTABLE INAR(1) MODELS
}

Márton Ispány, Gyula Pap, Martien C. A. van Zuijlen

Report No. 0109 (April 2001)

DEPARTMENT OF MATHEMATICS

UNIVERSITY OF NIJMEGEN

Toernooiveld

6525 ED Nijmegen

The Netherlands 


\title{
Asymptotic inference for nearly unstable INAR(1) models
}

\author{
Márton Ispány, Gyula Pap*, Martien C. A. van Zuijlen**
}

\begin{abstract}
The first-order integer-valued autoregressive (INAR(1)) process is investigated, where the autoregressive coefficient is close to one. It is shown that the limiting distribution of the conditional least-squares estimator for this coefficient is normal and, in contrast to the familiar $\operatorname{AR}(1)$ process, the rate of convergence is $n^{3 / 2}$. Finally, the nearly critical Galton-Watson process with unobservable immigration is discussed.
\end{abstract}

Keywords. Discrete time series, INAR(1) model, stable and nearly unstable models, conditional least squares estimator, asymptotic distribution, GaltonWatson process.

\section{Introduction}

In many practical situations we have to deal with non-negative integer-valued time series. Examples of these time series, known as counting processes, arise in several fields of medicine (see, e.g., Cardinal et.al. [5] and Franke and Seligmann [10]). To model counting processes Al-Osh and Alzaid [1] proposed a particular class of models, the so-called INAR(1) model. Later Al-Osh and Alzaid [2], Du and Li [9] and Latour [14] generalized this model by introducing the $\operatorname{INAR}(p)$ and $\operatorname{GINAR}(p)$ models. These processes can be considered as discrete analogues of the scalar- and

\footnotetext{
* Institute of Mathematics and Informatics, University of Debrecen, Pf. 12, H-4010 Debrecen, Hungary

** Department of Mathematics, University of Nijmegen, Toernooiveld 1, 6525 ED Nijmegen, The Netherlands

This research has been supported by NWO, The Netherlands (Nederlandse Organisatie voor Wetenschappelijk Onderzoek), by the Hungarian Ministry of Culture and Education under Grant No. FKFP 0121/1999, and by the Hungarian Foundation for Scientific Researchers under Grant No. OTKA-T032361/2000.
} 
vector-valued $\mathrm{AR}(p)$ processes, because their correlation structure is similar to that of the continuous-valued model (see (1.1)).

The present paper seems to be the first attempt to deal with the so-called nearly unstable INAR(1) model. For this model the autoregressive coefficient $\alpha_{n}$ is close to one, more precisely, $\alpha_{n}=1-\gamma_{n} / n$, where $\gamma_{n} \rightarrow \gamma \geqslant 0$. This parametrization has been suggested by Chan and Wei [6] for the usual AR(1) model. The main motivation of our investigation comes from econometrics, where the so-called 'unit root problem' plays an important role (see, e.g., the monograph of Tanaka [17]). We prove in Proposition 4.1 that the random step function defined by the nearly unstable INAR(1) process under the standard normalization converges to a continuous Gaussian martingale, which satisfies a stochastic differential equation similar to that of the Ornstein-Uhlenbeck process. By the help of this approximation it is shown that the conditional least squares estimate for the autoregressive coefficient is asymptotically normal.

To define the INAR(1) model let us recall the definition of the $\alpha \circ$ operator which is due to Steutel and van Harn [16].

1.1 Definition. Let $\left(Y_{j}\right)_{j \in \mathbb{N}}$ be a sequence of independent and identically distributed (i.i.d.) Bernoulli random variables with mean $\alpha$, independent of $X$, a non-negative integer-valued random variable. The Steutel and van Harn operator $\alpha \circ$ is defined by

$$
\alpha \circ X:= \begin{cases}\sum_{j=1}^{X} Y_{j}, & X>0, \\ 0, & X=0 .\end{cases}
$$

The sequence $\left(Y_{j}\right)_{j \in \mathbb{N}}$ is called a counting sequence.

The zero start INAR(1) time series model is defined as

$$
\left\{\begin{array}{l}
X_{k}=\alpha \circ X_{k-1}+\varepsilon_{k}, \quad k=1,2, \ldots, \\
X_{0}=0
\end{array}\right.
$$

where $\left(\varepsilon_{k}\right)_{k \in \mathbb{N}}$ is an i.i.d. sequence of non-negative integer-valued random variables with mean $\mu_{\varepsilon}$ and variance $\sigma_{\varepsilon}^{2}$, and the counting sequences $\left(Y_{j}\right)_{j \in \mathbb{N}}$ involved in $\alpha \circ X_{k-1}$ for $k=1,2, \ldots$ are mutually independent and independent of $\left(\varepsilon_{k}\right)_{k \in \mathbb{N}}$.

We have

$$
\mathrm{E} X_{k}=\alpha \mathrm{E} X_{k-1}+\mu_{\varepsilon}, \quad k=1,2, \ldots,
$$

hence

$$
\mathrm{E} X_{k}= \begin{cases}\frac{1-\alpha^{k}}{1-\alpha} \mu_{\varepsilon}, & \alpha \in[0,1), \quad \text { for } k \geqslant 0 . \\ k \mu_{\varepsilon}, & \alpha=1\end{cases}
$$


Moreover, by the recursion

$$
\operatorname{Var} X_{k}=\alpha^{2} \operatorname{Var} X_{k-1}+\alpha(1-\alpha) \mathrm{E} X_{k-1}+\sigma_{\varepsilon}^{2}, \quad k=1,2, \ldots,
$$

we obtain the variance structure

$$
\operatorname{Var} X_{k}=\left\{\begin{array}{ll}
\frac{1-\alpha^{2 k}}{1-\alpha^{2}} \sigma_{\varepsilon}^{2}+\frac{\left(\alpha-\alpha^{k}\right)\left(1-\alpha^{k}\right)}{1-\alpha^{2}} \mu_{\varepsilon}, & \alpha \in[0,1), \\
k \sigma_{\varepsilon}^{2}, & \alpha=1
\end{array} \quad \text { for } k \geqslant 0\right.
$$

Finally by the recursion

$$
\operatorname{Cov}\left(X_{k}, X_{\ell}\right)=\alpha \operatorname{Cov}\left(X_{k}, X_{\ell-1}\right), \quad 0 \leqslant k<\ell,
$$

we conclude

$$
\operatorname{Cov}\left(X_{k}, X_{\ell}\right)=\alpha^{|k-\ell|} \operatorname{Var} X_{k \wedge \ell} \quad \text { for } k, \ell \geqslant 0 .
$$

Hence for $\alpha \in[0,1)$ we have

$$
\lim _{k \rightarrow \infty} \mathrm{E} X_{k}=\frac{1}{1-\alpha} \mu_{\varepsilon}, \quad \lim _{k \rightarrow \infty} \operatorname{Var} X_{k}=\frac{1}{1-\alpha^{2}} \sigma_{\varepsilon}^{2}+\frac{\alpha}{1-\alpha^{2}} \mu_{\varepsilon} .
$$

The case $\alpha \in[0,1)$ is called stable or asymptotically stationary. The case $\alpha=1$ is called unstable.

Denote by $\mathcal{F}_{k}$ the $\sigma$-algebra generated by the random variables $X_{0}, X_{1}, \ldots, X_{k}$. Clearly $\left(X_{k}\right)_{k \in \mathbb{Z}_{+}}$is a Markov process, and we have

$$
\mathrm{E}\left(X_{k} \mid \mathcal{F}_{k-1}\right)=\mathrm{E}\left(X_{k} \mid X_{k-1}\right)=\alpha X_{k-1}+\mu_{\varepsilon}
$$

Thus the conditional least squares estimator (CLSE) $\widehat{\alpha}_{n}$ of $\alpha$ based on the observations $\left(X_{k}\right)_{1 \leqslant k \leqslant n}$ can be obtained by minimizing the sum of squares

$$
\sum_{k=1}^{n}\left(X_{k}-\alpha X_{k-1}-\mu_{\varepsilon}\right)^{2}
$$

and it has the form

$$
\widehat{\alpha}_{n}=\frac{\sum_{k=1}^{n} X_{k-1}\left(X_{k}-\mu_{\varepsilon}\right)}{\sum_{k=1}^{n} X_{k-1}^{2}} .
$$

In the stable case under the assumption $\mathrm{E} \varepsilon_{1}^{3}<+\infty$ we have

$$
n^{1 / 2}\left(\widehat{\alpha}_{n}-\alpha\right) \stackrel{\mathcal{D}}{\longrightarrow} \mathcal{N}\left(0, \sigma^{2}\right), \quad \sigma^{2}=\frac{\alpha(1-\alpha) \mathrm{E} X^{3}+\sigma_{\varepsilon}^{2} \mathrm{E} X^{2}}{\left(\mathrm{E} X^{2}\right)^{2}},
$$

where the distribution of $X$ is the common distribution of the unique stationary solution of the stable INAR(1) model

$$
X_{k}=\alpha \circ X_{k-1}+\varepsilon_{k}, \quad k \in \mathbb{Z} .
$$


(Obviously, $\mathrm{E} X^{2}$ and $\mathrm{E} X^{3}$ can be expressed by $\mu_{\varepsilon}, \sigma_{\varepsilon}^{2}, \mathrm{E} \varepsilon^{3}$ and $\alpha$ ). It easy to see that $\mathrm{E} X^{3}$ is finite iff the noise $\varepsilon$ has finite third moment. The convergence in (1.2) for the stationary solution of (1.3) follows from the decomposition

$n^{1 / 2}\left(\widehat{\alpha}_{n}-\alpha\right)=\frac{n^{-1 / 2} \sum_{k=1}^{n} X_{k-1}\left(\alpha \circ X_{k-1}-\alpha X_{k-1}\right)}{n^{-1} \sum_{k=1}^{n} X_{k-1}^{2}}+\frac{n^{-1 / 2} \sum_{k=1}^{n} X_{k-1}\left(\varepsilon_{k}-\mu_{\varepsilon}\right)}{n^{-1} \sum_{k=1}^{n} X_{k-1}^{2}}$

by standard martingale convergence argument, see Hall and Heyde [11] or Klimko and Nelson [13]. We should remark that the conditional variance of the numerator of the first term is given by

$$
\frac{1}{n} \sum_{k=1}^{n} \mathrm{E}\left(X_{k-1}^{2}\left(\alpha \circ X_{k-1}-\alpha X_{k-1}\right)^{2} \mid \mathcal{F}_{k-1}\right)=\frac{\alpha(1-\alpha)}{n} \sum_{k=1}^{n} X_{k-1}^{3} .
$$

Hence, by ergodicity of the stable INAR(1) model proved in $\mathrm{Du}$ and $\mathrm{Li}$ [9], it converges to a finite constant iff the third moment of $X$ is finite.

For the unstable case, it follows from our main result (Theorem 2.1) that

$$
n^{3 / 2}\left(\widehat{\alpha}_{n}-1\right) \stackrel{\mathcal{D}}{\longrightarrow} \mathcal{N}\left(0,3 \sigma_{\varepsilon}^{2} / \mu_{\varepsilon}^{2}\right) .
$$

In this case it is enough to assume the existence of a finite second moment, which follows from the fact that the first term of (1.4) vanishes and we have

$$
n^{3 / 2}\left(\widehat{\alpha}_{n}-1\right)=\frac{n^{-3 / 2} \sum_{k=1}^{n} X_{k-1}\left(\varepsilon_{k}-\mu_{\varepsilon}\right)}{n^{-3} \sum_{k=1}^{n} X_{k-1}^{2}} .
$$

\section{Main results for the nearly unstable INAR(1) model}

Let us consider the sequence of nearly unstable zero start INAR(1) models

$$
\left\{\begin{array}{l}
X_{k}^{(n)}=\alpha_{n} \circ X_{k-1}^{(n)}+\varepsilon_{k}^{(n)}, \quad k=1,2, \ldots \\
X_{0}^{(n)}=0
\end{array}\right.
$$

where $n=1,2, \ldots$,

$$
\alpha_{n}=1-\frac{\gamma_{n}}{n}, \quad \gamma_{n} \geqslant 0, \quad \gamma_{n} \rightarrow \gamma \geqslant 0,
$$

$\left(\varepsilon_{k}^{(n)}\right)_{k \in \mathbb{N}}$ are i.i.d. sequences of non-negative integer-valued random variables with mean $\mu_{\varepsilon}$ and variance $\sigma_{\varepsilon}^{2}$, and the counting sequences $\left(Y_{j}\right)_{j \in \mathbb{N}}$ involved in $\alpha_{n} \circ X_{k-1}^{(n)}$ are mutually independent and independent of $\left(\varepsilon_{k}^{(n)}\right)_{k \in \mathbb{N}}$. Let $\widehat{\alpha}_{n}$ be the CLSE of 
$\alpha$ based on the observations $\left(X_{k}^{(n)}\right)_{1 \leqslant k \leqslant n}$. We are interested in the asymptotic behaviour of the sequence $\left(\widehat{\alpha}_{n}\right)_{n \in \mathbb{N}}$. We have

$$
\widehat{\alpha}_{n}-\alpha_{n}=\frac{\sum_{k=1}^{n} X_{k-1}^{(n)}\left(X_{k}^{(n)}-\mu_{\varepsilon}-\alpha_{n} X_{k-1}^{(n)}\right)}{\sum_{k=1}^{n}\left(X_{k-1}^{(n)}\right)^{2}}=\frac{\sum_{k=1}^{n} X_{k-1}^{(n)} M_{k}^{(n)}}{\sum_{k=1}^{n}\left(X_{k-1}^{(n)}\right)^{2}}
$$

where

$$
M_{k}^{(n)}:=\alpha_{n} \circ X_{k-1}^{(n)}-\alpha_{n} X_{k-1}^{(n)}+\varepsilon_{k}^{(n)}-\mu_{\varepsilon} .
$$

We remark that

$$
M_{k}^{(n)}=X_{k}^{(n)}-\alpha_{n} X_{k-1}^{(n)}-\mu_{\varepsilon}=X_{k}^{(n)}-\mathrm{E}\left(X_{k}^{(n)} \mid \mathcal{F}_{k-1}^{(n)}\right),
$$

where $\mathcal{F}_{k}^{(n)}$ denotes the $\sigma$-algebra generated by the random variables $X_{0}^{(n)}, \ldots, X_{k}^{(n)}$. Let us introduce the random step functions

$$
X^{(n)}(t):=X_{[n t]}^{(n)}, \quad M^{(n)}(t):=\sum_{k=1}^{[n t]} M_{k}^{(n)}, \quad t \geqslant 0 .
$$

Then

$$
\widehat{\alpha}_{n}-\alpha_{n}=\frac{\int_{0}^{1} X^{(n)}(t) \mathrm{d} M^{(n)}(t)}{n \int_{0}^{1}\left(X^{(n)}(t)\right)^{2} \mathrm{~d} t} .
$$

By investigating the asymptotic behaviour of the sequences of stochastic processes $\left(X^{(n)}\right)_{n \in \mathbb{N}}, \quad\left(M^{(n)}\right)_{n \in \mathbb{N}}$ we obtain asymptotic normality of $\widehat{\alpha}_{n}$, namely

2.1 Theorem. We have

$$
n^{3 / 2}\left(\widehat{\alpha}_{n}-\alpha_{n}\right) \stackrel{\mathcal{D}}{\longrightarrow} \mathcal{N}\left(0, \sigma_{\gamma, \varepsilon}^{2}\right),
$$

where

$$
\sigma_{\gamma, \varepsilon}^{2}:= \begin{cases}\frac{\gamma^{2}\left(\sigma_{\varepsilon}^{2} \int_{0}^{1}\left(1-\mathrm{e}^{-\gamma t}\right)^{2} \mathrm{~d} t+\mu_{\varepsilon} \int_{0}^{1}\left(1-\mathrm{e}^{-\gamma t}\right)^{3} \mathrm{~d} t\right)}{\mu_{\varepsilon}^{2}\left(\int_{0}^{1}\left(1-\mathrm{e}^{-\gamma t}\right)^{2} \mathrm{~d} t\right)^{2}}, & \gamma>0, \\ \mu_{\varepsilon}^{2}, & \gamma=0 .\end{cases}
$$

We note that the purely unstable case $\alpha_{n}=1, n=1,2, \ldots$, is also included, where we have $\gamma_{n}=0, n=1,2, \ldots$, thus $\gamma=0$ and we obtain the asymptotic normality (1.5) in contrast to the usual purely unstable $\mathrm{AR}(1)$ case (see, e.g., White [19]; see also the discussion in Arató, Pap and Zuijlen [3]).

Finally, we should remark that, as pointed out by Dion et.al. [8] and Franke and Seligmann [10], the INAR(1) process is a special case of the Galton-Watson process with immigration. However, in the branching process framework it is assumed that 
the immigration component $\varepsilon_{k}$ is observed, which is not the case in our setup. We recall that a Galton-Watson process is said to be sub-critical if the expectation of the offspring distribution is less than one. Following our previous investigation, a GaltonWatson process is called nearly critical if the expectation of the offspring distribution is $\alpha_{n}=1-\gamma_{n} / n$, where $\gamma_{n} \rightarrow \gamma \geqslant 0$. Thus our main theorem can be reformulated as follows.

2.2 Corollary. Consider a nearly critical Galton-Watson process with Bernoulli offspring distribution and unobservable immigration with expectation $\mu_{\varepsilon}>0$ and variance $\sigma_{\varepsilon}^{2}<\infty$. Then the limiting distribution of the CLSE of the parameter of the offspring distribution is normal with variance (2.1).

We remark that the asymtotic normality in the sub-critical case with general offspring distribution and observed immigration is proved by Venkataraman and Nanthi [18]. The rate of convergence is $n^{1 / 2}$ in this case. We conjeture that our result can be extended for Galton-Watson processes with more general offspring distribution.

\section{Limit behaviour of $\left(M^{(n)}\right)_{n \in \mathbb{N}}$}

Clearly, we have $\mathrm{EM}^{(n)}(t)=0$. Moreover,

$$
\operatorname{Cov}\left(M_{k}^{(n)}, M_{\ell}^{(n)}\right)= \begin{cases}\sigma_{\varepsilon}^{2}+\alpha_{n}\left(1-\alpha_{n}\right) \mathrm{E} X_{k-1}^{(n)}, & k=\ell, \\ 0, & k \neq \ell,\end{cases}
$$

hence

$$
\begin{aligned}
\operatorname{Cov}\left(M^{(n)}(s), M^{(n)}(t)\right) & =\sum_{k=1}^{[n s] \wedge[n t]} \operatorname{Var}\left(M_{k}^{(n)}\right) \\
& =([n s] \wedge[n t]) \sigma_{\varepsilon}^{2}+\alpha_{n}\left(1-\alpha_{n}\right) \sum_{k=1}^{[n s] \wedge[n t]} \operatorname{E} X_{k-1}^{(n)} \\
& =([n s] \wedge[n t]) \sigma_{\varepsilon}^{2}+\alpha_{n} \mu_{\varepsilon} \sum_{k=1}^{[n s] \wedge[n t]}\left(1-\alpha_{n}^{k-1}\right) \\
& =([n s] \wedge[n t]) \sigma_{\varepsilon}^{2}+\alpha_{n}\left(([n s] \wedge[n t])-\frac{1-\alpha_{n}^{[n s] \wedge[n t]}}{1-\alpha_{n}}\right) \mu_{\varepsilon} .
\end{aligned}
$$

Moreover, $\alpha_{n}$ can be written in the form

$$
\alpha_{n}=\mathrm{e}^{-\gamma_{n}^{*} / n}, \quad \gamma_{n}^{*}>0, \quad \gamma_{n}^{*} \rightarrow \gamma \geqslant 0,
$$


thus

$$
\operatorname{Cov}\left(M^{(n)}(s), M^{(n)}(t)\right) \approx n T_{M}(s \wedge t)
$$

where

$$
T_{M}(t):= \begin{cases}\sigma_{\varepsilon}^{2} t+\mu_{\varepsilon}\left(t-\frac{1-\mathrm{e}^{-\gamma t}}{\gamma}\right), & \gamma>0, \\ \sigma_{\varepsilon}^{2} t, & \gamma=0 .\end{cases}
$$

We prove that the normalized process converges to a Gaussian process in distribution.

\subsection{Proposition. Let}

$$
\widetilde{M}^{(n)}(t):=\frac{M^{(n)}(t)}{\sqrt{n}}, \quad t \geqslant 0
$$

Then

$$
\widetilde{M}^{(n)} \stackrel{\mathcal{D}}{\longrightarrow} M \quad \text { as } n \rightarrow \infty
$$

in the Skorokhod space $\mathbb{D}\left(\mathbb{R}_{+}, \mathbb{R}\right)$, where $(M(t))_{t \geqslant 0}$ is a Gaussian process with zero mean and

$$
\operatorname{Cov}(M(s), M(t))=T_{M}(s \wedge t) .
$$

We remark that $(M(t))_{t \geqslant 0}$ is a continuous Gaussian process with independent (but not stationary) increments. Hence it is a continuous martingale, so that we can define stochastic integral with respect to it.

Proof. Let us introduce the random variables

$$
\xi_{k}^{(n)}=\alpha_{n} \circ X_{k-1}^{(n)}-\alpha_{n} X_{k-1}^{(n)}, \quad k=1, \ldots, n, n=1,2, \ldots
$$

In the sequel, we shall frequently use that $\mathrm{E}\left(\xi_{k}^{(n)} \mid \mathcal{F}_{k-1}^{(n)}\right)$ has a centered binomial distribution with parameters $X_{k-1}^{(n)}$ and $\alpha_{n}$. Thus,

$$
\mathrm{E}\left(\xi_{k}^{(n)} \mid \mathcal{F}_{k-1}^{(n)}\right)=0, \quad \mathrm{E}\left(\left(\xi_{k}^{(n)}\right)^{2} \mid \mathcal{F}_{k-1}^{(n)}\right)=\alpha_{n}\left(1-\alpha_{n}\right) X_{k-1}^{(n)} .
$$

Moreover, $\xi_{k}^{(n)}$ and $\varepsilon_{k}^{(n)}$ are independent. Since

$$
\mathrm{E}\left(M_{k}^{(n)} \mid \mathcal{F}_{k-1}^{(n)}\right)=\sum_{j=1}^{X_{k-1}^{(n)}} \mathrm{E}\left(Y_{j}-\alpha_{n}\right)+\mathrm{E}\left(\varepsilon_{k}^{(n)}-\mu_{\varepsilon}\right)=0,
$$

$\left(M_{k}^{(n)}\right)_{1 \leqslant k \leqslant n}$ is a martingale difference with respect to the filtration $\left(\mathcal{F}_{k}^{(n)}\right)_{0 \leqslant k \leqslant n}$ for all $n=1,2, \ldots$ Hence, it enough to verify that the conditional variances of the 
martingale difference array $\left(M_{k}^{(n)}, \mathcal{F}_{k}^{(n)}\right)_{k, n}$ tends to the variance function of the limit process in probability, i.e.

$$
\frac{1}{n} \sum_{k=1}^{[n t]} \mathrm{E}\left(\left(M_{k}^{(n)}\right)^{2} \mid \mathcal{F}_{k-1}^{(n)}\right) \stackrel{\mathrm{P}}{\longrightarrow} T_{M}(t)
$$

for all $t \geqslant 0$, and to prove that the conditional Lindeberg condition

$$
\frac{1}{n} \sum_{k=1}^{[n t]} \mathrm{E}\left(\left(M_{k}^{(n)}\right)^{2}\left\{\left|M_{k}^{(n)}\right|>\delta \sqrt{n}\right\} \mid \mathcal{F}_{k-1}^{(n)}\right) \stackrel{\mathrm{P}}{\longrightarrow} 0
$$

holds for all $\delta>0, t \geqslant 0$ (see Jacod and Shiryaev [12, Theorem VIII.3.33]).

In order to prove (3.2) we note that

$$
\mathrm{E}\left(\left(M_{k}^{(n)}\right)^{2} \mid \mathcal{F}_{k-1}^{(n)}\right)=\mathrm{E}\left(\left(\xi_{k}^{(n)}\right)^{2} \mid \mathcal{F}_{k-1}^{(n)}\right)+\mathrm{E}\left(\varepsilon_{k}^{(n)}-\mu_{\varepsilon}\right)^{2}=\alpha_{n}\left(1-\alpha_{n}\right) X_{k-1}^{(n)}+\sigma_{\varepsilon}^{2} .
$$

Bearing in mind the definition of $T_{M}(t)$ (see (3.1)) it is enough to show that

$$
\frac{1}{n} \sum_{k=1}^{[n t]} \mathrm{E}\left(\left(\xi_{k}^{(n)}\right)^{2} \mid \mathcal{F}_{k-1}^{(n)}\right)=\frac{\alpha_{n}\left(1-\alpha_{n}\right)}{n} \sum_{k=1}^{[n t]} X_{k-1}^{(n)} \stackrel{\mathrm{P}}{\longrightarrow} \mu_{\varepsilon}\left(t-\frac{1-\mathrm{e}^{-\gamma t}}{\gamma}\right),
$$

where the right hand side is defined as zero in case $\gamma=0$. One can see that

$$
\frac{\alpha_{n}\left(1-\alpha_{n}\right)}{n} \sum_{k=1}^{[n t]} \mathrm{E} X_{k-1}^{(n)}=\frac{\alpha_{n}\left(1-\alpha_{n}\right)}{n} \sum_{k=1}^{[n t]} \frac{1-\alpha_{n}^{k-1}}{1-\alpha_{n}} \mu_{\varepsilon}=\alpha_{n} \mu_{\varepsilon}\left(\frac{[n t]}{n}-\frac{1-\alpha_{n}^{[n t]}}{n\left(1-\alpha_{n}\right)}\right)
$$

tends to the right hand side of (3.4). Applying the Chebysev's inequality

$$
\mathrm{P}\left(\left|\frac{1}{n} \sum_{k=1}^{[n t]}\left(\mathrm{E}\left(\left(\xi_{k}^{(n)}\right)^{2} \mid \mathcal{F}_{k-1}^{(n)}\right)-\mathrm{E}\left(\xi_{k}^{(n)}\right)^{2}\right)\right|>\delta\right) \leqslant \frac{1}{\delta^{2}} \operatorname{Var}\left(\frac{\alpha_{n}\left(1-\alpha_{n}\right)}{n} \sum_{k=1}^{[n t]} X_{k-1}^{(n)}\right)
$$

for any $\delta>0$, it remains to show that the right hand side converges to zero. We obtain

$$
\begin{aligned}
& \operatorname{Var}\left(\frac{\alpha_{n}\left(1-\alpha_{n}\right)}{n} \sum_{k=1}^{[n t]} X_{k-1}^{(n)}\right)=\frac{\alpha_{n}^{2}\left(1-\alpha_{n}\right)^{2}}{n^{2}} \sum_{k, \ell=1}^{[n t]} \alpha_{n}^{|k-\ell|} \operatorname{Var}\left(X_{k \wedge \ell-1}\right) \\
& \quad=\frac{\alpha_{n}^{2}\left(1-\alpha_{n}\right)^{2}}{n^{2}}\left(\sum_{k=1}^{[n t]} \operatorname{Var}\left(X_{k-1}^{(n)}\right)+2 \sum_{k=1}^{[n t]-1}\left(\alpha_{n}+\ldots+\alpha_{n}^{[n t]-k}\right) \operatorname{Var}\left(X_{k-1}^{(n)}\right)\right),
\end{aligned}
$$


where the second sum is defined as zero in case $t<2 / n$. Here, for the first term we have

$$
\begin{aligned}
& \frac{\alpha_{n}^{2}\left(1-\alpha_{n}\right)^{2}}{n^{2}} \sum_{k=1}^{[n t]} \operatorname{Var}\left(X_{k-1}^{(n)}\right) \\
& =\frac{\alpha_{n}^{2}\left(1-\alpha_{n}\right)}{n^{2}\left(1+\alpha_{n}\right)} \sum_{k=1}^{[n t]}\left(1-\alpha_{n}^{2(k-1)}\right) \sigma_{\varepsilon}^{2}+\left(\alpha_{n}-\alpha_{n}^{k-1}-\alpha_{n}^{k}+\alpha_{n}^{2(k-1)}\right) \mu_{\varepsilon} \\
& =\frac{\alpha_{n}^{2}\left(1-\alpha_{n}\right)}{n\left(1+\alpha_{n}\right)}\left(\frac{[n t]}{n}\left(\sigma_{\varepsilon}^{2}+\alpha_{n} \mu_{\varepsilon}\right)-\frac{\left(1+\alpha_{n}\right)\left(1-\alpha_{n}^{[n t]}\right)}{n\left(1-\alpha_{n}\right)} \mu_{\varepsilon}+\frac{1-\alpha_{n}^{2[n t]}}{n\left(1-\alpha_{n}^{2}\right)}\left(\mu_{\varepsilon}-\sigma_{\varepsilon}^{2}\right)\right),
\end{aligned}
$$

which is approximately

$$
\frac{\gamma}{2 n^{2}}\left(\left(\sigma_{\varepsilon}^{2}+\mu_{\varepsilon}\right) t-2 \frac{1-\mathrm{e}^{-\gamma t}}{\gamma} \mu_{\varepsilon}+\frac{1-\mathrm{e}^{-2 \gamma t}}{2 \gamma}\left(\mu_{\varepsilon}-\sigma_{\varepsilon}^{2}\right)\right)
$$

for large $n$, i.e., it tends to zero. For the second term we have

$$
\begin{aligned}
& \frac{2 \alpha_{n}^{2}\left(1-\alpha_{n}\right)^{2}}{n^{2}} \sum_{k=1}^{[n t]-1}\left(\alpha_{n}+\ldots+\alpha_{n}^{[n t]-k}\right) \operatorname{Var}\left(X_{k-1}^{(n)}\right) \\
= & \frac{2 \alpha_{n}^{3}}{n^{2}\left(1+\alpha_{n}\right)} \sum_{k=1}^{[n t]-1}\left(1-\alpha_{n}^{[n t]-k}\right)\left(\left(1-\alpha_{n}^{2(k-1)}\right) \sigma_{\varepsilon}^{2}+\left(\alpha_{n}-\alpha_{n}^{k-1}-\alpha_{n}^{k}+\alpha_{n}^{2(k-1)}\right) \mu_{\varepsilon}\right) \\
= & \frac{2 \alpha_{n}^{3}}{n\left(1+\alpha_{n}\right)}\left(\frac{[n t]-1}{n}\left(\sigma_{\varepsilon}^{2}+\alpha_{n} \mu_{\varepsilon}\right)-\frac{\left(1+\alpha_{n}\right)\left(1-\alpha_{n}^{[n t]-1}\right)}{n\left(1-\alpha_{n}\right)} \mu_{\varepsilon}+\frac{1-\alpha_{n}^{2[n t]-2}}{n\left(1-\alpha_{n}^{2}\right)}\left(\mu_{\varepsilon}-\sigma_{\varepsilon}^{2}\right)\right. \\
& \left.-\alpha_{n} \frac{1-\alpha_{n}^{[n t]-1}}{n\left(1-\alpha_{n}\right)}\left(\sigma_{\varepsilon}^{2}+\alpha_{n} \mu_{\varepsilon}\right)+\frac{[n t]-1}{n} \alpha_{n}^{[n t]-1}\left(1+\alpha_{n}\right) \mu_{\varepsilon}+\alpha_{n}^{[n t]-1} \frac{1-\alpha_{n}^{[n t]-1}}{n\left(1-\alpha_{n}\right)}\left(\sigma_{\varepsilon}^{2}-\mu_{\varepsilon}\right)\right),
\end{aligned}
$$

which is approximately

$$
\frac{1}{n}\left(\left(\sigma_{\varepsilon}^{2}+3 \mu_{\varepsilon}\right) t-4 \frac{1-\mathrm{e}^{-\gamma t}}{\gamma} \mu_{\varepsilon}+\frac{1-\mathrm{e}^{-2 \gamma t}}{2 \gamma}\left(\mu_{\varepsilon}-\sigma_{\varepsilon}^{2}\right)\right)
$$

for large $n$, i.e., it tends to zero.

Proving the conditional Lindeberg condition (3.3) we note that for any pair $\xi, \eta$ of random variables

$$
\{|\xi+\eta|>\delta\} \leqslant\{|\xi|>\delta / 2\}+\{|\eta|>\delta / 2\}
$$


for all $\delta>0$. Hence, it is enough to show the following:

$$
\begin{array}{r}
\frac{1}{n} \sum_{k=1}^{[n t]} \mathrm{E}\left(\left(\xi_{k}^{(n)}\right)^{2}\left\{\left|\xi_{k}^{(n)}\right|>\delta \sqrt{n}\right\} \mid \mathcal{F}_{k-1}^{(n)}\right) \stackrel{\mathrm{P}}{\longrightarrow} 0, \\
\frac{1}{n} \sum_{k=1}^{[n t]} \mathrm{E}\left(\left(\xi_{k}^{(n)}\right)^{2}\left\{\left|\varepsilon_{k}^{(n)}-\mu_{\varepsilon}\right|>\delta \sqrt{n}\right\} \mid \mathcal{F}_{k-1}^{(n)}\right) \stackrel{\mathrm{P}}{\longrightarrow} 0, \\
\frac{1}{n} \sum_{k=1}^{[n t]} \mathrm{E}\left(\left(\varepsilon_{k}^{(n)}-\mu_{\varepsilon}\right)^{2}\left\{\left|\xi_{k}^{(n)}\right|>\delta \sqrt{n}\right\} \mid \mathcal{F}_{k-1}^{(n)}\right) \stackrel{\mathrm{P}}{\longrightarrow} 0, \\
\frac{1}{n} \sum_{k=1}^{[n t]} \mathrm{E}\left(\left(\varepsilon_{k}^{(n)}-\mu_{\varepsilon}\right)^{2}\left\{\left|\varepsilon_{k}^{(n)}-\mu_{\varepsilon}\right|>\delta \sqrt{n}\right\}\right)
\end{array}
$$

for all $\delta>0$. To prove the convergence (3.6) we apply the Cauchy-Schwarz and the Markov inequalities in the conditional form obtaining

$$
\begin{aligned}
\mathrm{E}\left(\left(\xi_{k}^{(n)}\right)^{2}\left\{\left|\xi_{k}^{(n)}\right|>\delta \sqrt{n}\right\} \mid \mathcal{F}_{k-1}^{(n)}\right) & \leqslant\left(\mathrm{E}\left(\left(\xi_{k}^{(n)}\right)^{4} \mid \mathcal{F}_{k-1}^{(n)}\right) \cdot \mathrm{P}\left(\left|\xi_{k}^{(n)}\right|>\delta \sqrt{n} \mid \mathcal{F}_{k-1}^{(n)}\right)\right)^{1 / 2} \\
& \leqslant \frac{1}{\delta \sqrt{n}}\left(\mathrm{E}\left(\left(\xi_{k}^{(n)}\right)^{4} \mid \mathcal{F}_{k-1}^{(n)}\right) \cdot \mathrm{E}\left(\left(\xi_{k}^{(n)}\right)^{2} \mid \mathcal{F}_{k-1}^{(n)}\right)\right)^{1 / 2}
\end{aligned}
$$

Note that the centered fourth moment of a binomial distribution $\xi$ with parameters $n$ and $p$ is given by

$$
\mathrm{E}(\xi-\mathrm{E} \xi)^{4}=n p(1-p)(3(n-2) p(1-p)+1) .
$$

Thus, the non-negative random variable on the left hand side of (3.6) can be majorized by

$$
\begin{aligned}
& \frac{\alpha_{n}\left(1-\alpha_{n}\right)}{\delta n^{3 / 2}} \sum_{k=1}^{[n t]} X_{k-1}^{(n)}\left(3 X_{k-1}^{(n)} \alpha_{n}\left(1-\alpha_{n}\right)+1\right)^{1 / 2} \\
& \leqslant \frac{\alpha_{n}\left(1-\alpha_{n}\right)}{\delta n^{3 / 2}}\left(\sum_{k=1}^{[n t]}\left(X_{k-1}^{(n)}\right)^{2} \cdot\left([n t]+3 \alpha_{n}\left(1-\alpha_{n}\right) \sum_{k=1}^{[n t]} X_{k-1}^{(n)}\right)\right)^{1 / 2} .
\end{aligned}
$$

We know that $n\left(1-\alpha_{n}\right)$ tends to $\gamma$ and by (3.4)

$$
\frac{[n t]}{n}+\frac{3 \alpha_{n}\left(1-\alpha_{n}\right)}{n} \sum_{k=1}^{[n t]} X_{k-1}^{(n)} \stackrel{\mathrm{P}}{\longrightarrow} t+3\left(t-\frac{1-\mathrm{e}^{-\gamma t}}{\gamma}\right) .
$$

It remains to show that

$$
\frac{1}{n^{4}} \sum_{k=1}^{[n t]}\left(X_{k-1}^{(n)}\right)^{2} \stackrel{\mathrm{P}}{\longrightarrow} 0
$$


But, this fact follows by Markov's inequality, since

$$
\begin{aligned}
& \mathrm{P}\left(\frac{1}{n^{4}} \sum_{k=1}^{[n t]}\left(X_{k-1}^{(n)}\right)^{2}>\delta\right) \leqslant \frac{1}{\delta n^{4}} \sum_{k=1}^{[n t]} \mathrm{E}\left(X_{k-1}^{(n)}\right)^{2}=\frac{1}{\delta n^{4}} \sum_{k=1}^{[n t]}\left(\left(\mathrm{E} X_{k-1}^{(n)}\right)^{2}+\operatorname{Var} X_{k-1}^{(n)}\right) \\
& \leqslant \frac{1}{\delta n^{4}}\left(\frac{1}{\left(1-\alpha_{n}\right)^{2}}\left([n t]-2 \frac{1-\alpha_{n}^{[n t]}}{1-\alpha_{n}}+\frac{1-\alpha_{n}^{2[n t]}}{1-\alpha_{n}^{2}}\right) \mu_{\varepsilon}^{2}\right. \\
& \left.\quad+\frac{1}{1-\alpha_{n}^{2}}\left([n t]\left(\sigma_{\varepsilon}^{2}+\alpha_{n} \mu_{\varepsilon}\right)-\frac{\left(1+\alpha_{n}\right)\left(1-\alpha_{n}^{[n t]}\right)}{1-\alpha_{n}} \mu_{\varepsilon}+\frac{1-\alpha_{n}^{2[n t]}}{\left(1-\alpha_{n}^{2}\right.}\left(\mu_{\varepsilon}-\sigma_{\varepsilon}^{2}\right)\right)\right)
\end{aligned}
$$

which is approximately

$$
\begin{cases}\frac{1}{\delta \gamma^{2} n}\left(t-2 \frac{1-\mathrm{e}^{-\gamma t}}{\gamma}+\frac{1-\mathrm{e}^{2 \gamma t}}{2 \gamma}\right) \mu_{\varepsilon}^{2}, & \gamma>0 \\ \frac{t^{3}}{3 \delta n} \mu_{\varepsilon}^{2}, & \gamma=0\end{cases}
$$

i.e., it tends to zero. By the independence of $\xi_{k}^{(n)}$ and $\varepsilon_{k}^{(n)}$ and Chebysev's inequality we have that the non-negative random variable defined in (3.7) is less than the non-negative random variable

$$
\frac{1}{n} \sum_{k=1}^{[n t]} \mathrm{E}\left(\left(\xi_{k}^{(n)}\right)^{2} \mid \mathcal{F}_{k-1}^{(n)}\right) \cdot \frac{\sigma_{\varepsilon}^{2}}{\delta^{2} n} .
$$

We have already proved that the first term goes to a finite limit depending on $t$ in probability (see (3.4)), hence the desired convergence follows. Similarly, by independence, the random variable defined by (3.8) equals to

$$
\frac{\sigma_{\varepsilon}^{2}}{n} \sum_{k=1}^{[n t]}\left\{\left|\xi_{k}^{(n)}\right|>\delta \sqrt{n}\right\} .
$$

We prove that this random variable tends to zero in mean square as $n \rightarrow \infty$. By the Cauchy-Schwarz and the Chebysev inequalities we obtain

$$
\begin{aligned}
& \mathrm{E}\left(\frac{\sigma_{\varepsilon}^{2}}{n} \sum_{k=1}^{[n t]}\left\{\left|\xi_{k}^{(n)}\right|>\delta \sqrt{n}\right\}\right)^{2} \leqslant \frac{\sigma_{\varepsilon}^{4}}{n^{2}} \sum_{k, \ell=1}^{[n t]}\left(\mathrm{P}\left(\left|\xi_{k}^{(n)}\right|>\delta \sqrt{n}\right) \mathrm{P}\left(\left|\xi_{\ell}^{(n)}\right|>\delta \sqrt{n}\right)\right)^{1 / 2} \\
& \leqslant \frac{\sigma_{\varepsilon}^{4}}{\delta^{2} n^{3}} \sum_{k, \ell=1}^{[n t]}\left(\mathrm{E}\left(\xi_{k}^{(n)}\right)^{2} \mathrm{E}\left(\xi_{\ell}^{(n)}\right)^{2}\right)^{1 / 2} \leqslant \frac{\sigma_{\varepsilon}^{4} \alpha_{n}\left(1-\alpha_{n}\right)}{\delta^{2} n^{3}}\left(\sum_{k=1}^{[n t]}\left(\mathrm{E} X_{k-1}^{(n)}\right)^{1 / 2}\right)^{2} \\
& \leqslant \frac{\sigma_{\varepsilon}^{4} \alpha_{n}\left(1-\alpha_{n}\right)[n t]}{\delta^{2} n^{3}} \sum_{k=1}^{[n t]} \mathrm{E} X_{k-1}^{(n)},
\end{aligned}
$$


which converges to zero by (3.5). Finally, (3.9) is evident since $\operatorname{Var} \varepsilon_{k}=\sigma_{\varepsilon}^{2}$ is finite.

In case $\gamma=0$ the process $M / \sigma_{\varepsilon}$ is a standard Wiener process. Comparing the covariance structures one can show easily that the process $M$ can be represented in the form

$$
M(t)=W\left(T_{M}(t)\right), \quad t \geqslant 0 .
$$

Note that Proposition 1 can be reformulated in the following manner: the sequence of normalized processes $\left(\widetilde{M}^{(n)}\right)_{n \in \mathbb{N}}$ converges to the process $\left(W\left(T_{M}(t)\right)\right)_{t} \geqslant 0$ given by a time changed standard Wiener process $(W(t))_{t \geqslant 0}$ in the Skorokhod space $\mathbb{D}\left(\mathbb{R}_{+}, \mathbb{R}\right)$. (See Rootzén [15], Theorem 3.5.)

We remark that the function $T_{M}$ can be written also in the form

$$
T_{M}(t)=\int_{0}^{t}\left(\sigma_{\varepsilon}^{2}+\mu_{\varepsilon}\left(1-\mathrm{e}^{-\gamma u}\right)\right) \mathrm{d} u .
$$

Comparing again the covariance structures we obtain another representation of the process $(M(t))_{t \geqslant 0}$ in the form

$$
M(t)=\int_{0}^{t} h_{M}(s) \mathrm{d} W(s), \quad t \geqslant 0,
$$

where

$$
h_{M}(t):=\sqrt{T_{M}^{\prime}(t)}=\sqrt{\sigma_{\varepsilon}^{2}+\mu_{\varepsilon}\left(1-\mathrm{e}^{-\gamma t}\right)} .
$$

Consequently, the process $(M(t))_{t \geqslant 0}$ is the unique solution of the stochastic differential equation (SDE)

$$
\left\{\begin{array}{l}
\mathrm{d} M(t)=h_{M}(t) \mathrm{d} W(t), \quad t \geqslant 0, \\
M(0)=0
\end{array}\right.
$$

\section{Continuous approximation of nearly unstable INAR processes}

In this section we investigate the limiting process of the sequence of random step functions $\left(X^{(n)}(t)\right)_{t \geqslant 0}, \quad n=1,2, \ldots$, induced by the nearly unstable $\operatorname{INAR}(1)$ process in the Skorokhod space.

We have

$$
\mathrm{E}^{(n)}(t)=\frac{1-\alpha_{n}^{[n t]}}{1-\alpha_{n}} \mu_{\varepsilon}=\frac{1-\mathrm{e}^{-\gamma_{n}^{*}[n t] / n}}{1-\mathrm{e}^{-\gamma_{n}^{*} / n}} \mu_{\varepsilon} \approx n \mu_{X}(t),
$$

where

$$
\mu_{X}(t):= \begin{cases}\frac{1-\mathrm{e}^{-\gamma t}}{\gamma} \mu_{\varepsilon}=\mu_{\varepsilon} \int_{0}^{t} e^{-\gamma u} \mathrm{~d} u, & \gamma>0, \\ t \mu_{\varepsilon}, & \gamma=0,\end{cases}
$$


and similarly

$$
\begin{aligned}
& \operatorname{Cov}\left(X^{(n)}(s), X^{(n)}(t)\right) \\
& =\alpha_{n}^{|[n s]-[n t]|}\left(\frac{1-\alpha_{n}^{2([n s] \wedge[n t])}}{1-\alpha_{n}^{2}} \sigma_{\varepsilon}^{2}+\frac{\left(\alpha_{n}-\alpha_{n}^{[n s] \wedge[n t]}\right)\left(1-\alpha_{n}^{[n s] \wedge[n t]}\right)}{1-\alpha_{n}^{2}} \mu_{\varepsilon}\right) \approx n \varrho_{X}(s, t),
\end{aligned}
$$

where

$$
\varrho_{X}(s, t):= \begin{cases}\mathrm{e}^{-\gamma|s-t|}\left(\frac{1-\mathrm{e}^{-2 \gamma(s \wedge t)}}{2 \gamma} \sigma_{\varepsilon}^{2}+\frac{\left(1-\mathrm{e}^{-\gamma(s \wedge t)}\right)^{2}}{2 \gamma} \mu_{\varepsilon}\right), & \gamma>0, \\ (s \wedge t) \sigma_{\varepsilon}^{2}, & \gamma=0 .\end{cases}
$$

We prove that the normalized process converges again to a Gaussian process in distribution.

4.1 Proposition. Let

$$
\tilde{X}^{(n)}(t):=\frac{X^{(n)}(t)-\mathrm{E} X^{(n)}(t)}{\sqrt{n}}, \quad t \geqslant 0 .
$$

Then

$$
\left(\widetilde{M}^{(n)}, \widetilde{X}^{(n)}\right) \stackrel{\mathcal{D}}{\longrightarrow}(M, X) \quad \text { as } n \rightarrow \infty
$$

in the Skorokhod space $\mathbb{D}\left(\mathbb{R}_{+}, \mathbb{R}^{2}\right)$, where

$$
X(t):=\int_{0}^{t} \mathrm{e}^{-\gamma(t-s)} \mathrm{d} M(s), \quad t \geqslant 0 .
$$

Moreover, $(X(t))_{t \geqslant 0}$ is a continuous Gaussian martingale with zero mean and

$$
\operatorname{Cov}(X(s), X(t))=\varrho_{X}(s, t), \quad s, t \geqslant 0 .
$$

We remark that $(X(t))_{t \geqslant 0}$ does not have independent increments.

We shall need a simple lemma, which gives a sufficient condition for convergence to a functional of a continuous process. The proof is based on the Continuous Mapping Theorem (see Billingsley [4, Theorem 5.5]), and it can be found in Arató, Pap and Zuijlen [3].

For measurable mappings $\Phi, \Phi_{n}: \mathbb{D}\left(\mathbb{R}_{+}, \mathbb{R}^{k}\right) \rightarrow \mathbb{D}\left(\mathbb{R}_{+}, \mathbb{R}^{\ell}\right), \quad n=1,2, \ldots$ we shall write $\Phi_{n} \rightsquigarrow \Phi$ if $\left\|\Phi_{n}\left(x_{n}\right)-\Phi(x)\right\|_{\infty} \rightarrow 0$ for all $x, x_{n} \in \mathbb{D}\left(\mathbb{R}_{+}, \mathbb{R}^{k}\right)$ with $\left\|x_{n}-x\right\|_{\infty} \rightarrow 0$, where $\|\cdot\|_{\infty}$ denotes the the supremum norm.

4.2 Lemma. Let $\Phi, \Phi_{n}: \mathbb{D}\left(\mathbb{R}_{+}, \mathbb{R}^{k}\right) \rightarrow \mathbb{D}\left(\mathbb{R}_{+}, \mathbb{R}^{\ell}\right), \quad n=1,2, \ldots$ be measurable mappings such that $\Phi_{n} \rightsquigarrow \Phi$. Let $Z, Z_{n}, n=1,2, \ldots$ be stochastic processes with values in $\mathbb{D}\left(\mathbb{R}_{+}, \mathbb{R}^{k}\right)$ such that $Z_{n} \stackrel{\mathcal{D}}{\longrightarrow} Z$ in $\mathbb{D}\left(\mathbb{R}_{+}, \mathbb{R}^{k}\right)$ and almost all trajectories of $Z$ are continuous. Then, $\Phi_{n}\left(Z_{n}\right) \stackrel{\mathcal{D}}{\longrightarrow} \Phi(Z)$ in $\mathbb{D}\left(\mathbb{R}_{+}, \mathbb{R}^{\ell}\right)$. 
Proof of Proposition 4.1. The purpose of the following discussion is to show that there exist measurable mappings $\Phi_{n}: \mathbb{D}\left(\mathbb{R}_{+}, \mathbb{R}\right) \rightarrow \mathbb{D}\left(\mathbb{R}_{+}, \mathbb{R}^{2}\right), \quad n=1,2, \ldots$, such that $\left(\widetilde{M}^{(n)}, \widetilde{X}^{(n)}\right)=\Phi_{n}\left(\widetilde{M}^{(n)}\right)$. We have

$$
M_{k}^{(n)}=X_{k}^{(n)}-\alpha_{n} X_{k-1}^{(n)}-\mu_{\varepsilon}=\left(X_{k}^{(n)}-\mathrm{E} X_{k}^{(n)}\right)-\alpha_{n}\left(X_{k-1}^{(n)}-\mathrm{E} X_{k-1}^{(n)}\right),
$$

which implies

$$
X_{k}^{(n)}-\mathrm{E} X_{k}^{(n)}=\sum_{j=1}^{k} \alpha_{n}^{k-j} M_{j}^{(n)}
$$

Consequently

$$
\begin{aligned}
\widetilde{X}^{(n)}(t) & =\frac{1}{\sqrt{n}} \sum_{j=1}^{[n t]} \alpha_{n}^{[n t]-j} M_{j}^{(n)}=\sum_{j=1}^{[n t]} \alpha_{n}^{[n t]-j}\left(\widetilde{M}^{(n)}\left(\frac{j}{n}\right)-\widetilde{M}^{(n)}\left(\frac{j-1}{n}\right)\right) \\
& =\widetilde{M}^{(n)}\left(\frac{[n t]}{n}\right)-\sum_{j=1}^{[n t]-1}\left(\alpha_{n}^{[n t]-j-1}-\alpha_{n}^{[n t]-j}\right) \widetilde{M}^{(n)}\left(\frac{j}{n}\right) \\
& =\widetilde{M}^{(n)}\left(\frac{[n t]}{n}\right)-\sum_{j=1}^{[n t]-1}\left(\mathrm{e}^{-\gamma_{n}^{*}([n t]-j-1) / n}-\mathrm{e}^{-\gamma_{n}^{*}([n t]-j) / n}\right) \widetilde{M}^{(n)}\left(\frac{j}{n}\right) \\
& =\widetilde{M}^{(n)}\left(\frac{[n t]}{n}\right)-\gamma_{n}^{*} \sum_{j=1}^{[n t]-1} \int_{j / n}^{(j+1) / n} \mathrm{e}^{-\gamma_{n}^{*}([n t] / n-s)} \mathrm{d} s \widetilde{M}^{(n)}\left(\frac{j}{n}\right) \\
& =\widetilde{M}^{(n)}\left(\frac{[n t]}{n}\right)-\gamma_{n}^{*} \int_{0}^{[n t] / n} \mathrm{e}^{-\gamma_{n}^{*}([n t] / n-s)} \widetilde{M}^{(n)}(s) \mathrm{d} s,
\end{aligned}
$$

which implies $\left(\widetilde{M}^{(n)}, \widetilde{X}^{(n)}\right)=\Phi_{n}\left(\widetilde{M}^{(n)}\right)$ with

$$
\Phi_{n}(x)(t)=\left(x(t), x\left(\frac{[n t]}{n}\right)-\gamma_{n}^{*} \int_{0}^{[n t] / n} \mathrm{e}^{-\gamma_{n}^{*}([n t] / n-s)} x(s) \mathrm{d} s\right) .
$$

Clearly $\Phi_{n} \rightsquigarrow \Phi$, where

$$
\Phi(x)(t)=\left(x(t), x(t)-\gamma \int_{0}^{t} \mathrm{e}^{-\gamma(t-s)} x(s) \mathrm{d} s\right) .
$$

Itô's formula yields

$$
\int_{0}^{t} \mathrm{e}^{-\gamma(t-s)} \mathrm{d} M(s)=M(t)-\gamma \int_{0}^{t} \mathrm{e}^{-\gamma(t-s)} M(s) \mathrm{d} s,
$$

hence $\left(\widetilde{M}^{(n)}, \widetilde{X}^{(n)}\right) \stackrel{\mathcal{D}}{\longrightarrow}(M, X)$ by Proposition 3.1 and Lemma 4.2. 
In fact, in case $\gamma=0$ the process $X / \sigma_{\varepsilon}$ is a standard Wiener process. In case $\gamma>0$ the covariance function of the process $X$ can be written also in the form

$$
\operatorname{Cov}(X(s), X(t))=\frac{\mathrm{e}^{-\gamma|s-t|}-\mathrm{e}^{-\gamma(s+t)}}{2 \gamma} \sigma_{\varepsilon}^{2}+\frac{\left(\mathrm{e}^{-\gamma|s-t| / 2}-\mathrm{e}^{-\gamma(s+t) / 2}\right)^{2}}{2 \gamma} \mu_{\varepsilon},
$$

where the first term is the covariance function of the zero start Ornstein-Uhlenbeck process $(Y(t))_{t} \geqslant 0$ defined by the SDE

$$
\left\{\begin{array}{l}
\mathrm{d} Y(t)=-\gamma Y(t) \mathrm{d} t+\sigma_{\varepsilon} \mathrm{d} W(t), \quad t \geqslant 0 \\
Y(0)=0
\end{array}\right.
$$

or by

$$
Y(t)=\sigma_{\varepsilon} \int_{0}^{t} \mathrm{e}^{\gamma(u-t)} \mathrm{d} W(u)
$$

Comparing the covariance structures one can show easily that the process $(X(t))_{t} \geqslant 0$ can be represented in the form

$$
X(t)=\mathrm{e}^{-\gamma t} W\left(T_{X}(t)\right), \quad t \geqslant 0
$$

where

$$
T_{X}(t)= \begin{cases}\frac{\mathrm{e}^{2 \gamma t}-1}{2 \gamma} \sigma_{\varepsilon}^{2}+\frac{\left(\mathrm{e}^{\gamma t}-1\right)^{2}}{2 \gamma} \mu_{\varepsilon}, & \gamma>0 \\ \sigma_{\varepsilon}^{2}, & \gamma=0\end{cases}
$$

We remark that the function $T_{X}$ can be written also in the form

$$
T_{X}(t)=\int_{0}^{t} \mathrm{e}^{2 \gamma u}\left(\sigma_{\varepsilon}^{2}+\mu_{\varepsilon}\left(1-\mathrm{e}^{-\gamma u}\right)\right) \mathrm{d} u
$$

We also have another representation in the form

$$
X(t)=\mathrm{e}^{-\gamma t} \int_{0}^{t} h_{X}(s) \mathrm{d} W(s), \quad t \geqslant 0
$$

where

$$
h_{X}(t):=\sqrt{T_{X}^{\prime}(t)}=\mathrm{e}^{\gamma t} h_{M}(t)=\mathrm{e}^{\gamma t} \sqrt{\sigma_{\varepsilon}^{2}+\mu_{\varepsilon}\left(1-\mathrm{e}^{-\gamma t}\right)} .
$$

Consequently, the process $(X(t))_{t} \geqslant 0$ is the unique solution of the stochastic differential equation (SDE)

$$
\left\{\begin{array}{l}
\mathrm{d} X(t)=-\gamma X(t) \mathrm{d} t+\sqrt{\sigma_{\varepsilon}^{2}+\mu_{\varepsilon}\left(1-\mathrm{e}^{-\gamma t}\right)} \mathrm{d} W(t), \quad t \geqslant 0 \\
X(0)=0
\end{array}\right.
$$


It can be also written in the form

$$
\left\{\begin{array}{l}
\mathrm{d} X(t)=-\gamma X(t) \mathrm{d} t+\mathrm{d} M(t), \quad t \geqslant 0 \\
X(0)=0
\end{array}\right.
$$

By formulae (4.1) and (4.3) we can think of the process $(X(t))_{t \geqslant 0}$ as a generalized Ornstein-Uhlenbeck process governed by the continuous Gaussian martingale $(M(t))_{t \geqslant 0}$.

\section{Asymptotic behaviour of the CLSE of the coeffi- cient}

We have

$$
\frac{1}{n} \int_{0}^{1} X^{(n)}(t) \mathrm{d} M^{(n)}(t)=\int_{0}^{1}\left(\widetilde{X}^{(n)}(t)+\frac{1}{\sqrt{n}} \mathrm{E} X^{(n)}(t)\right) \mathrm{d} \widetilde{M}^{(n)}(t)=A_{n}+B_{n} \sqrt{n},
$$

where

$$
A_{n}:=\int_{0}^{1} \widetilde{X}^{(n)}(t) \mathrm{d} \widetilde{M}^{(n)}(t), \quad B_{n}:=\frac{1}{n} \int_{0}^{1} \mathrm{E} X^{(n)}(t) \mathrm{d} \widetilde{M}^{(n)}(t) .
$$

Moreover

$$
\frac{1}{n} \int_{0}^{1}\left(X^{(n)}(t)\right)^{2} \mathrm{~d} t=\int_{0}^{1}\left(\tilde{X}^{(n)}(t)+\frac{1}{\sqrt{n}} \mathrm{E} X^{(n)}(t)\right)^{2} \mathrm{~d} t=C_{n}+D_{n} \sqrt{n}+E_{n} n,
$$

where

$$
\begin{gathered}
C_{n}:=\int_{0}^{1}\left(\widetilde{X}^{(n)}(t)\right)^{2} \mathrm{~d} t, \quad D_{n}:=\frac{2}{n} \int_{0}^{1} \widetilde{X}^{(n)}(t) \mathrm{E} X^{(n)}(t) \mathrm{d} t \\
E_{n}:=\frac{1}{n^{2}} \int_{0}^{1}\left(\mathrm{E} X^{(n)}(t)\right)^{2} \mathrm{~d} t .
\end{gathered}
$$

5.1 Proposition. We have

(i) $A_{n} \stackrel{\mathcal{D}}{\longrightarrow} \int_{0}^{1} X(t) \mathrm{d} M(t)=: A$ ，

(ii) $B_{n} \stackrel{\mathcal{D}}{\longrightarrow} \int_{0}^{1} \mu_{X}(t) \mathrm{d} M(t)=: B$,

(iii) $C_{n} \stackrel{\mathcal{D}}{\longrightarrow} \int_{0}^{1} X(t)^{2} \mathrm{~d} t=: C$ ，

(iv) $D_{n} \stackrel{\mathcal{D}}{\longrightarrow} 2 \int_{0}^{1} \mu_{X}(t) X(t) \mathrm{d} t=: D$ 
(v) $E_{n} \rightarrow \int_{0}^{1} \mu_{X}(t)^{2} \mathrm{~d} t=: E$.

Proof. (i). First we remark that formula (4.2) yields

$$
M_{k}^{(n)}=\alpha_{n}^{k}\left(V_{k}^{(n)}-V_{k-1}^{(n)}\right),
$$

where

$$
V_{k}^{(n)}:=\alpha_{n}^{-k}\left(X_{k}^{(n)}-\mathrm{E} X_{k}^{(n)}\right) .
$$

Hence

$$
\begin{aligned}
A_{n} & =\frac{1}{n} \sum_{k=1}^{n}\left(X_{k-1}^{(n)}-\mathrm{E} X_{k-1}^{(n)}\right) M_{k}^{(n)}=\frac{1}{n} \sum_{k=1}^{n} \alpha_{n}^{2 k-1} V_{k-1}^{(n)}\left(V_{k}^{(n)}-V_{k-1}^{(n)}\right) \\
& =\frac{1}{2 n} \sum_{k=1}^{n} \alpha_{n}^{2 k-1}\left(\left(V_{k}^{(n)}\right)^{2}-\left(V_{k-1}^{(n)}\right)^{2}\right)-\frac{1}{2 n} \sum_{k=1}^{n} \alpha_{n}^{2 k-1}\left(V_{k}^{(n)}-V_{k-1}^{(n)}\right)^{2} \\
& =A_{n}^{(1)}+A_{n}^{(2)}-A_{n}^{(3)},
\end{aligned}
$$

where

$$
\begin{aligned}
A_{n}^{(1)} & :=\frac{1}{2 n} \alpha_{n}^{2 n-1}\left(V_{n}^{(n)}\right)^{2}=\frac{1}{2 n \alpha_{n}}\left(X_{n}^{(n)}-\mathrm{E} X_{n}^{(n)}\right)^{2}=\frac{1}{2 \alpha_{n}}\left(\widetilde{X}^{(n)}(1)\right)^{2}, \\
A_{n}^{(2)} & :=\frac{1-\alpha_{n}^{2}}{2 n} \sum_{k=1}^{n} \alpha_{n}^{2 k-3}\left(V_{k-1}^{(n)}\right)^{2}=\frac{1-\alpha_{n}^{2}}{2 n \alpha_{n}} \sum_{k=1}^{n}\left(X_{k-1}^{(n)}-\mathrm{E} X_{k-1}^{(n)}\right)^{2} \\
& =\frac{\left(1+\alpha_{n}\right) \gamma_{n}}{2 n \alpha_{n}} \sum_{k=1}^{n}\left(\widetilde{X}^{(n)}\left(\frac{k-1}{n}\right)\right)^{2}=\frac{\left(1+\alpha_{n}\right) \gamma_{n}}{2 \alpha_{n}} \int_{0}^{1}\left(\widetilde{X}^{(n)}(s)\right)^{2} \mathrm{~d} s, \\
A_{n}^{(3)} & :=\frac{1}{2 n \alpha_{n}} \sum_{k=1}^{n}\left(M_{k}^{(n)}\right)^{2} .
\end{aligned}
$$

Applying Proposition 4.1 and the Continuous Mapping Theorem we obtain

$$
A_{n}^{(1)}+A_{n}^{(2)} \stackrel{\mathcal{D}}{\longrightarrow} \frac{1}{2}(X(1))^{2}+\gamma \int_{0}^{1}(X(s))^{2} \mathrm{~d} s .
$$

The convergences (3.2) and (3.3) imply

$$
\frac{1}{n} \sum_{k=1}^{[n t]}\left(M_{k}^{(n)}\right)^{2} \stackrel{\mathrm{P}}{\longrightarrow} T_{M}(t)
$$

for all $t \in \mathbb{R}_{+}$(see again Jacod and Shiryaev [12, Theorem VIII.3.33]), consequently,

$$
A_{n}^{(3)} \stackrel{\mathrm{P}}{\longrightarrow} \frac{1}{2} T_{M}(1) .
$$


We note that for sequences $\left(\xi_{n}\right)_{n \in \mathbb{N}},\left(\eta_{n}\right)_{n \in \mathbb{N}}$ and $\left(\zeta_{n}\right)_{n \in \mathbb{N}}$ with $\xi_{n} \stackrel{\mathcal{D}}{\longrightarrow} \xi, \eta_{n} \stackrel{\mathrm{P}}{\longrightarrow} a$ and $\zeta_{n} \stackrel{\mathrm{P}}{\longrightarrow} b$, where $a, b$ are finite constants, we have $\xi_{n} \eta_{n}+\zeta_{n} \stackrel{\mathcal{D}}{\longrightarrow} a \xi+b$ (see Slutsky's theorem and its corollary in Chow and Teicher [7, 8.1]). Hence

$$
A_{n}^{(1)}+A_{n}^{(2)}-A_{n}^{(3)} \stackrel{\mathcal{D}}{\longrightarrow} \frac{1}{2}(X(1))^{2}+\gamma \int_{0}^{1}(X(s))^{2} \mathrm{~d} s-\frac{1}{2} T_{M}(1) .
$$

Let

$$
V(t):=\mathrm{e}^{\gamma t} X(t)=\int_{0}^{t} \mathrm{e}^{\gamma s} \sqrt{\sigma_{\varepsilon}^{2}+\mu_{\varepsilon}\left(1-\mathrm{e}^{-\gamma s}\right)} \mathrm{d} W(s), \quad t \in \mathbb{R}_{+} .
$$

Then $(V(t))_{t \geqslant 0}$ is a continuous Gaussian process with independent (but not stationary) increments, hence it is a continuous martingale. Its stochastic differential has the form

$$
\mathrm{d} V(t)=\mathrm{e}^{\gamma t} \sqrt{\sigma_{\varepsilon}^{2}+\mu_{\varepsilon}\left(1-\mathrm{e}^{-\gamma t}\right)} \mathrm{d} W(t)
$$

Moreover

$$
A=\int_{0}^{1} X(t) \mathrm{d} M(t)=\int_{0}^{1} \mathrm{e}^{-2 \gamma t} V(t) \mathrm{d} V(t)
$$

Itô's formula implies

$$
\mathrm{d}\left(V(t)^{2}\right)=2 V(t) \mathrm{d} V(t)+\mathrm{e}^{2 \gamma t}\left(\sigma_{\varepsilon}^{2}+\mu_{\varepsilon}\left(1-\mathrm{e}^{-\gamma t}\right)\right) \mathrm{d} t
$$

hence

$$
\int_{0}^{1} \mathrm{e}^{-2 \gamma t} V(t) \mathrm{d} V(t)=\frac{1}{2} \int_{0}^{1} \mathrm{e}^{-2 \gamma t} \mathrm{~d}\left(V(t)^{2}\right)-\frac{1}{2} \int_{0}^{1}\left(\sigma_{\varepsilon}^{2}+\mu_{\varepsilon}\left(1-\mathrm{e}^{-\gamma t}\right)\right) \mathrm{d} t .
$$

Again by Itô's formula

$$
\int_{0}^{1} \mathrm{e}^{-2 \gamma t} \mathrm{~d}\left(V(t)^{2}\right)=\mathrm{e}^{-2 \gamma t} V(1)^{2}+2 \gamma \int_{0}^{1} \mathrm{e}^{-2 \gamma t} V(t)^{2} \mathrm{~d} t=X(1)^{2}+2 \gamma \int_{0}^{1} X(t)^{2} \mathrm{~d} t
$$

hence finally by (3.10)

$$
A=\frac{1}{2}(X(1))^{2}+\gamma \int_{0}^{1}(X(s))^{2} \mathrm{~d} s-\frac{1}{2} T_{M}(1),
$$

and we obtain (i). 
(ii). We have

$$
\begin{aligned}
B_{n} & =\frac{1}{n^{3 / 2}} \sum_{k=1}^{n}\left(\mathrm{E} X_{k-1}^{(n)}\right) M_{k}^{(n)}=\frac{\mu_{\varepsilon}}{n^{3 / 2}} \sum_{k=1}^{n} \sum_{j=0}^{k-2} \alpha_{n}^{j} M_{k}^{(n)} \\
& =\frac{\mu_{\varepsilon}}{n^{3 / 2}} \sum_{j=0}^{n-2} \alpha_{n}^{j} \sum_{k=j+2}^{n} M_{k}^{(n)}=\frac{\mu_{\varepsilon}}{n^{3 / 2}} \sum_{j=0}^{n-2} \alpha_{n}^{j}\left(\sum_{k=1}^{n} M_{k}^{(n)}-\sum_{k=1}^{j+1} M_{k}^{(n)}\right) \\
& =\frac{\mu_{\varepsilon}}{n} \sum_{j=0}^{n-2} \mathrm{e}^{-\gamma_{n}^{*} j / n}\left(\widetilde{M}^{(n)}(1)-\widetilde{M}^{(n)}\left(\frac{j+1}{n}\right)\right) \\
& =\mu_{\varepsilon} \sum_{j=0}^{n-2} \mathrm{e}^{-\gamma_{n}^{*} j / n} \int_{(j+1) / n}^{(j+2) / n}\left(\widetilde{M}^{(n)}(1)-\widetilde{M}^{(n)}(t)\right) \mathrm{d} t=\Psi_{n}\left(\widetilde{M}^{(n)}\right),
\end{aligned}
$$

where the functionals $\Psi_{n}: \mathbb{D}\left(\mathbb{R}_{+}, \mathbb{R}\right) \rightarrow \mathbb{R}, n \in \mathbb{N}$, are defined by

$$
\Psi_{n}(x):=\mu_{\varepsilon} \sum_{j=0}^{n-2} \mathrm{e}^{-\gamma_{n}^{*} j / n} \int_{(j+1) / n}^{(j+2) / n}(x(1)-x(t)) \mathrm{d} t .
$$

We have $\left|\Psi_{n}\left(x_{n}\right)-\Psi(x)\right| \rightarrow 0$ for all $x, x_{n} \in \mathbb{D}\left(\mathbb{R}_{+}, \mathbb{R}\right)$ with $\left\|x_{n}-x\right\|_{\infty} \rightarrow 0$, where the functional $\Psi: \mathbb{D}\left(\mathbb{R}_{+}, \mathbb{R}\right) \rightarrow \mathbb{R}$ is defined by

$$
\Psi(x):=\mu_{\varepsilon} \int_{0}^{1} \mathrm{e}^{-\gamma t}(x(1)-x(t)) \mathrm{d} t .
$$

Hence Proposition 3.1 and an appropriate analogue of Lemma 4.2 imply

$$
B_{n} \stackrel{\mathcal{D}}{\longrightarrow} \mu_{\varepsilon} \int_{0}^{1} \mathrm{e}^{-\gamma t}(M(1)-M(t)) \mathrm{d} t
$$

Moreover by Itô's formula

$$
\begin{aligned}
B & =\int_{0}^{1} \mu_{X}(t) \mathrm{d} M(t)=\mu_{X}(1) M(1)-\int_{0}^{1} M(t) \mathrm{d} \mu_{X}(t) \\
& =M(1) \mu_{\varepsilon} \int_{0}^{1} \mathrm{e}^{-\gamma t} \mathrm{~d} t-\mu_{\varepsilon} \int_{0}^{1} M(t) \mathrm{e}^{-\gamma t} \mathrm{~d} t=\mu_{\varepsilon} \int_{0}^{1} \mathrm{e}^{-\gamma t}(M(1)-M(t)) \mathrm{d} t
\end{aligned}
$$

thus we obtain (ii).

(iii). The convergence $C_{n} \stackrel{\mathcal{D}}{\longrightarrow} C$ is an easy consequence of Proposition 4.1 and the Continuous Mapping Theorem.

(iv). We have

$$
\begin{aligned}
D_{n} & =\frac{2}{n^{2}} \sum_{k=1}^{n-1}\left(\mathrm{EX}_{k}^{(n)}\right) \widetilde{X}^{(n)}\left(\frac{k}{n}\right)=\frac{2 \mu_{\varepsilon}}{n^{2}\left(1-\alpha_{n}\right)} \sum_{k=1}^{n-1}\left(1-\alpha_{n}^{k-1}\right) \widetilde{X}^{(n)}\left(\frac{k}{n}\right) \\
& =\frac{2 \mu_{\varepsilon}}{\gamma_{n}} \sum_{k=1}^{n-1}\left(1-\mathrm{e}^{-\gamma_{n}^{*}(k-1) / n}\right) \int_{k / n}^{(k+1) / n} \widetilde{X}^{(n)}(t) \mathrm{d} t=\Psi_{n}\left(\widetilde{X}^{(n)}\right),
\end{aligned}
$$


where the functionals $\Psi_{n}: \mathbb{D}\left(\mathbb{R}_{+}, \mathbb{R}\right) \rightarrow \mathbb{R}, \quad n \in \mathbb{N}$ are defined by

$$
\Psi_{n}(x):=\frac{2 \mu_{\varepsilon}}{\gamma_{n}} \sum_{k=1}^{n-1}\left(1-\mathrm{e}^{-\gamma_{n}^{*}(k-1) / n}\right) \int_{k / n}^{(k+1) / n} x(t) \mathrm{d} t .
$$

We have $\left|\Psi_{n}\left(x_{n}\right)-\Psi(x)\right| \rightarrow 0$ for all $x, x_{n} \in \mathbb{D}\left(\mathbb{R}_{+}, \mathbb{R}\right)$ with $\left\|x_{n}-x\right\|_{\infty} \rightarrow 0$, where the functional $\Psi: \mathbb{D}\left(\mathbb{R}_{+}, \mathbb{R}\right) \rightarrow \mathbb{R}$ is defined by

$$
\Psi(x):=2 \int_{0}^{1} \mu_{X}(t) x(t) \mathrm{d} t
$$

Hence Proposition 4.1 and an appropriate analogue of Lemma 4.2 imply

$$
D_{n} \stackrel{\mathcal{D}}{\longrightarrow} 2 \int_{0}^{1} \mu_{X}(t) X(t) \mathrm{d} t=D
$$

(v). We have

$$
E_{n}=\frac{1}{n^{3}} \sum_{k=1}^{n-1}\left(\mathrm{E} X_{k}^{(n)}\right)^{2}=\frac{\mu_{\varepsilon}^{2}}{n^{3}\left(1-\alpha_{n}\right)^{2}} \sum_{k=1}^{n-1}\left(1-\alpha_{n}^{k}\right)^{2} \rightarrow \int_{0}^{1} \mu_{X}(t)^{2} \mathrm{~d} t=E
$$

Proof of Theorem 1. We have

$$
n^{3 / 2}\left(\widehat{\alpha}_{n}-\alpha_{n}\right)=\sqrt{n} \frac{A_{n}+B_{n} \sqrt{n}}{C_{n}+D_{n} \sqrt{n}+E_{n} n}=\frac{A_{n} / \sqrt{n}+B_{n}}{C_{n} / n+D_{n} / \sqrt{n}+E_{n}} .
$$

Applying again Lemma 5.1 and Slutsky's theorem we obtain

$$
\frac{A_{n}}{\sqrt{n}} \stackrel{\mathrm{P}}{\longrightarrow} 0, \quad \frac{C_{n}}{n} \stackrel{\mathrm{P}}{\longrightarrow} 0, \quad \frac{D_{n}}{\sqrt{n}} \stackrel{\mathrm{P}}{\longrightarrow} 0,
$$

and finally

$$
n^{3 / 2}\left(\widehat{\alpha}_{n}-\alpha_{n}\right) \stackrel{\mathcal{D}}{\longrightarrow} \frac{B}{E}=\frac{\int_{0}^{1} \mu_{X}(t) \mathrm{d} M(t)}{\int_{0}^{1} \mu_{X}(t)^{2} \mathrm{~d} t} .
$$

The limit distribution is clearly a normal law with zero mean. We have

$$
B=\int_{0}^{1} \mu_{X}(t) \mathrm{d} M(t)=\int_{0}^{1} \mu_{X}(t) \sqrt{\sigma_{\varepsilon}^{2}+\mu_{\varepsilon}\left(1-\mathrm{e}^{-\gamma t}\right)} \mathrm{d} W(t),
$$

hence

$$
E\left(\int_{0}^{1} \mu_{X}(t) \mathrm{d} M(t)\right)^{2}=\int_{0}^{1} \mu_{X}(t)^{2}\left(\sigma_{\varepsilon}^{2}+\mu_{\varepsilon}\left(1-\mathrm{e}^{-\gamma t}\right)\right) \mathrm{d} t
$$

and finally we obtain the variance of the limit law. 


\section{References}

[1] Al-Osh, M.A. and Alzaid, A.A. (1987). First-order integer-valued autoregressive (INAR(1)) process. J. Time Ser. Anal. 8, 261-275.

[2] Al-Osh, M.A. and Alzaid, A.A. (1990). An integer-valued $p$ th-order autoregressive structure $(\operatorname{INAR}(p))$ process. J. Appl. Prob. 27, 314-324.

[3] Arató, M., Pap, G. and Zuijlen, M.v. (2001). Asymptotic inference for spatial autoregression and orthogonality of Ornstein-Uhlenbeck sheets. To appear in Computers Math. Appl. 39.

[4] Billingsley, P. (1968). Convergence of Probability Measures. John Wiley \& Sons, New York.

[5] Cardinal, M., Roy, R. and Lambert, J. (1999). On the application of integervalued time series models for the analysis of disease incidence. Stat. Medicine 18, 2025-2039.

[6] Chan, N.H. and Wei, C.Z. (1987). Asymptotic inference for nearly nonstationary AR(1) processes. Ann. Statist. 15, 1050-1063.

[7] Chow, Y.S. and Teicher, H. (1978). Probability Theory. Springer-Verlag, New York, Heidelberg, Berlin.

[8] Dion, J.P., Gauthier, G. and Latour, A. (1995). Branching processes with immigration and integer-valued time series. Serdica Math. J. 21, 123-136.

[9] DU, J.G. and LI, Y. (1991). The integer-valued autoregressive INAR $(p)$ model. J. Time Ser. Anal. 12, 129-142.

[10] Franke, J. and Seligmann, T. (1993). Conditional maximum likelihood estimates for INAR(1) processes and their application to modeling epileptic seizure counts. in Subba Rao, T. (ed.), Developments in Time Series Analysis, Chapman and Hall, London, pp. 310-330.

[11] Hall, P. and Heyde, C.C. (1980). Martingale Limit Theory and its Application. Academic Press, New York.

[12] Jacod, J. and Shiryayev, A.N. (1987). Limit Theorems for Stochastic Processes. Springer-Verlag, Berlin, Heidelberg, New York, London, Paris, Tokyo.

[13] Klimko, L.A. and Nelson, P.I. (1978). On conditional least squares estimation for stochastic processes. Ann. Statist. 6, 629-642.

[14] Latour, A. (1997). The multivariate GINAR(p) process. Adv. Appl. Prob. 29, 228-248. 
[15] Rootzén, H. (1983). Central limit theory for martingales via random change of time. Probability and Mathematical Statistics. Essays in Hon. C.-G. Esseen. Uppsala, Sweden, 154-189.

[16] Steutel, F. and van Harn, K. (1979). Discrete analogues of selfdecomposability and stability. Ann. Probab. 7, 893-99.

[17] Tanaka, K. (1996). Time Series Analysis. Nonstationary and Noninvertible Distribution Theory. John Wiley \& Sons, New York.

[18] Venkataraman, K.N. and Nanthi, K. (1982). A limit theorem on subcritical Galton-Watson process with immigration. Ann. Probab. 10, 1069-1074.

[19] White, J. S. (1958). The limiting distribution of the serial correlation coefficient in the explosive case. Ann. Math. Statist. 29, 1188-1197. 